\section{Multifactor ANOVAs with and without repeated measures: Programs for limited microcomputer systems}

\section{ALLEN H. WOLACH \\ Illinois Institute of Technology, Chicago, Illinois 60616}

The present set of statistical programs is for microcomputer systems with limited capabilities. All of the programs will work in systems that have less than $8 \mathrm{~K}$ of user memory (RAM). The programs require a subset of the BASIC language that should be available in virtually all microcomputer systems. For example, the programs do not assume that a given microcomputer system can perform exponentiation. Similarly, the programs calculate square roots with a subroutine as opposed to assuming the microcomputer system has a square root (SQR) library function. Specifically, the programs require the following mathematical and relational operators: $+,-, *, \mid,=,\langle\rangle,,\langle$, and $\rangle=$. The only key words and commands required by the programs are DIM, END, FOR-NEXT, GOSU B, GOTO, IF-THEN, INPUT, LET, PRINT, RETURN, STOP, and TAB.

Provisional mean formulas (as opposed to computational formulas) are used in the programs. The provisional mean formulas can provide accurate results in systems that perform computations to only six decimal places.

Description. The programs are (1) independentgroups one-way analysis of variance, equal in required for each group (listing one page), (2) same as 1, but accepts unequal $\mathrm{n}$ and performs unweighted-means analysis of variance (one page), (3) two-way analysis of variance without replications (randomized-block analysis of variance), equal $n$ required for each treatment level (one page), (4) independent-groups two-way analysis of variance, equal $n$ required for each subgroup (two pages), (5) same as 4 , but accepts unequal $\mathrm{n}$ for subgroups and performs unweighted means analysis of variance (two pages), (6) split-plot two-way analysis of variance, two factors, equal n required for each treatment level (two pages), (7) randomized-block factorial analysis of variance, equal $n$ required for each subgroup (three pages), (8) independent-groups three-way analysis of variance, equal $n$ required for each subgroup (three pages), (9) same as 8 , but accepts unequal $n$ for subgroups and performs unweighted-means analysis of variance (four pages), and (10) split-plot three-way analysis of variance, equal $n$ required for each subgroup (four pages).

No programs require arrays with more than two dimensions. Programs 1 and 2 require no arrays. Programs 4 and 5 require only one single-subscripted array. The most complicated program (10) requires one singlesubscripted array and two double-subscripted arrays. In order to accommodate large data sets, data are not retained after they are entered and processed.

Input. The programs start by printing instructions for data entry on the monitor. Appropriate prompts appear on the monitor before data are entered (e.g., ENTER OBSERVATION 1, GROUP 2).

Output. The programs produce all output on the terminal monitor. Means and variances for all cells are printed out. Means for all interactions are also printed out. The resuits of the ANOVA are printed out in the form of an ANOVA summary table.

Availability. Listings for the programs can be obtained from Allen $\mathrm{H}$. Wolach, Department of Psychology, Illinois Institute of Technology, Chicago, Illinois 60616. Include the number(s) of the programs and 10 cents for each listing page (to cover photocopying and mailing). All 10 programs can be obtained for $\$ 2$.

(Accepted for publication April 28, 1981.) 